\title{
Statistical Replicability
}

Matthew B.A. McDermott, Shirly Wang, Nikki Marinsek, Rajesh Ranganath, Marzyeh Ghassemi, Luca Foschini

\section{Source}

Matthew B. A. McDermott, Shirly Wang, Nikki Marinsek, Rajesh Ranganath, Marzyeh

Ghassemi, Luca Foschini. (2019). Reproducibility in Machine Learning for Health.

arXiv:1907.01463v1 [Cs.LG]. https://arxiv.org/pdf/1907.01463.pdf

Statistical replicability refers to the ability of a result to hold under re-sampled conditions that yield different technical configurations, but should not statistically affect the claimed result (e.g., a different set of random seeds, or train/test splits). 